\title{
Investigating ballistic impact properties of woven kenaf-aramid hybrid composites
}

\begin{abstract}
In this study, the ballistic impact performance of woven kenaf-Kevlar hybrid and non-hybrid composites against fragment simulating projectiles (FSPs) was investigated. All the composites were prepared using the hand lay-up technique, method, followed by static load compression. The hybrid composites consist of Kevlar fabric and woven kenaf layers. The results obtained indicate that the energy absorption, ballistic limit velocity (V 50) and failure behaviour of the composites during the impact event were affected by the woven kenaf hybridisation. The additional kenaf layers in hybrid composites resulted in the increase in composites thickness and areal density, thus increased the energy absorption (14.46\% to $41.30 \%)$ and V 50 (5.5\% to $8.44 \%$ ). It was observed that the hybrid composites failed through a combination of fibre shear, delamination and fibre fracture in the impacted surface, woven kenaf-Kevlar interface and rear surface respectively. Although the specific energy absorption was lower for the hybrid composites, further investigations need to be carried out to utilise the great potential natural fibres.
\end{abstract}

Keyword: Aramid fibre; Ballistic impact; Hybrid composites; Laminates; Woven kenaf 